\title{
When does a generalized Boolean quasiring become a Boolean ring?
}

\author{
Ivan Chajda ${ }^{1} \cdot$ Helmut Länger ${ }^{1,2}$ \\ Published online: 19 December 2017 \\ (c) The Author(s) 2017. This article is an open access publication
}

\begin{abstract}
Generalized Boolean quasirings are ring-like structures used as algebraic models in the foundations of axiomatic quantum mechanics. The quantum mechanical system corresponding to such a quasiring turns out to be a classical one if and only if this quasiring is a Boolean ring with unit. We characterize this situation by a single identity.
\end{abstract}

Keywords Generalized Boolean quasiring · Boolean ring with unit · Lattice with an antitone involution · Axiomatic quantum mechanics

The classical approach to the foundations of axiomatic quantum mechanics was introduced by Mackey (2004). It was modified by D. Dorninger, G. Eigenthaler, M. Mączyński and both authors, see Chajda (1996), Chajda and Eigenthaler (1998), Chajda and Länger (2007), Chajda et al. (2004), Dorninger (1998), Dorninger et al. (1997a, b, 1999, 2000, 2001a,b), Länger (1998), Länger and Mączyński (2005). (For concepts concerning lattice theory we refer the reader to the monograph (Grätzer 2011).) They introduced several socalled ring-like structures used for modelling quantum logics. These ring-like structures are called Boolean quasirings, generalized Boolean quasirings and orthopseudorings (Chajda 1996; Chajda and Eigenthaler 1998) and their mutual relations were investigated by Chajda and Eigenthaler (1998).

It is known that for classical physics the corresponding logical structure are Boolean algebras which are in one-toone correspondence to Boolean rings with unit. Hence it is a natural question to find the difference between Boolean rings with unit and the mentioned ring-like structures. In the following we present an identity which has the property that

Communicated by A. Di Nola.

Helmut Länger

helmut.laenger@tuwien.ac.at

Ivan Chajda

ivan.chajda@upol.cz

1 Department of Algebra and Geometry, Faculty of Science, Palacký University Olomouc, 17. listopadu 12, 77146 Olomouc, Czech Republic

2 Faculty of Mathematics and Geoinformation, Institute of Discrete Mathematics and Geometry, TU Wien, Wiedner Hauptstraße 8-10, 1040 Vienna, Austria when added to the identities of a uniquely representable generalized Boolean quasiring converts this ring-like structure into a Boolean ring with unit.

Recall that a Boolean ring with unit is a unitary ring $\mathbf{R}=(R,+, \cdot, 0,1)$ that is idempotent, i.e. the identity $x x \approx x$ holds. (Here and in the following identities are written by using the symbol " $\approx$ " which means that the corresponding identity holds for arbitrary elements of the considered algebra(s).) It is well-known that every Boolean ring is commutative and of characteristic 2 , i.e. the identity $x+x \approx 0$ holds. The notion of a generalized Boolean quasiring was introduced in Dorninger et al. (1997a) as follows:

Definition 1 A generalized Boolean quasiring is an algebra $\mathbf{R}=(R,+, \cdot, 0,1)$ of type $(2,2,0,0)$ satisfying the following identities:

$$
\begin{aligned}
x+y & \approx y+x \\
x+0 & \approx x \\
(x y) z & \approx x(y z) \\
x y & \approx y x \\
x x & \approx x \\
x 0 & \approx 0 \\
x 1 & \approx x \\
1+(1+x y)(1+y) & \approx y
\end{aligned}
$$

The algebra $\mathbf{R}$ is called uniquely representable (cf. Dorninger 1998) if it satisfies the identity

$(1+(1+x)(1+y))(1+x y) \approx x+y$ 
and a Boolean quasiring (cf. Dorninger et al. 1997a) if it satisfies identity (9) as well as the following identity:

$x+x \approx 0$

Remark 2 Identity (1) follows from identities (4) and (9). This means that commutativity of addition follows from unique representability of the corresponding generalized Boolean quasiring.

In Dorninger et al. (1997a) the following result was proved:

Theorem 3 A Boolean quasiring $\mathbf{R}=(R,+, \cdot, 0,1)$ is a Boolean ring with unit if and only if it satisfies the identity

$x(1+y) \approx x+x y$.

Recall that a bounded lattice with an antitone involution is an algebra $\mathbf{L}=\left(L, \vee, \wedge,{ }^{\prime}, 0,1\right)$ such that $(L, \vee, \wedge, 0,1)$ is a bounded lattice and the following identities are satisfied:

$$
\begin{gathered}
(x \vee y)^{\prime} \approx x^{\prime} \wedge y^{\prime}, \\
(x \wedge y)^{\prime} \approx x^{\prime} \vee y^{\prime}, \\
\left(x^{\prime}\right)^{\prime} \approx x .
\end{gathered}
$$

The following well-known result (cf. Dorninger et al. 1997a) shows that every uniquely representable generalized Boolean quasiring can be assigned to a bounded lattice with an antitone involution and conversely.

\section{Proposition 4 The formulas}

$x \vee y:=1+(1+x)(1+y), x \wedge y:=x y$ and $x^{\prime}:=1+x$

and

$x+y:=(x \vee y) \wedge\left(x^{\prime} \vee y^{\prime}\right)$ and $x y:=x \wedge y$

induce a one-to-one correspondence between uniquely representable generalized Boolean quasirings and bounded lattices with an antitone involution.

The aim of the present paper is to generalize Theorem 3 for generalized Boolean quasirings as follows:

Theorem 5 A uniquely representable generalized Boolean quasiring $\mathbf{R}=(R,+, \cdot, 0,1)$ is a Boolean ring with unit if and only if it satisfies the identity

$x(1+x y) \approx x(1+y)$.

Remark 6 Identities (3), (5) and (11) together imply (12). This means that Theorem 5 is in fact stronger than Theorem 3 .
We start with some easy observations:

Lemma 7 (i) Identities (5) and (8) together imply the identities

$1+(1+x) \approx x$

and

$(1+x y)(1+y) \approx 1+y$.

(ii) Identities (2), (5) and (8) together imply the identity $1+1 \approx 0$.

(iii) Identities (2), (5), (6), (7), (8) and (12) together imply the identity $x(1+x) \approx 0$.

Proof (i) Identities (5) and (8) together imply $1+(1+x) \approx$ $1+(1+x)(1+x) \approx 1+(1+x x)(1+x) \approx x$ and hence $(1+x y)(1+y) \approx 1+(1+(1+x y)(1+y)) \approx 1+y$. (ii) Identity (2) and (i) together imply $1+1 \approx 1+(1+0) \approx$ 0 .

(iii) Identities (7), (12) and (6) and (ii) together imply $x(1+$ $x) \approx x(1+x 1) \approx x(1+1) \approx x 0 \approx 0$.

The most important property that discerns Boolean rings with unit from generalized Boolean quasirings is distributivity. In order to prove this property the following result will be useful:

Lemma 8 Every generalized Boolean quasiring $\mathbf{R}=$ $(R,+, \cdot, 0,1)$ satisfying identity (12) also satisfies the identity $(1+(1+x)(1+y)) z \approx 1+(1+x z)(1+y z)$.

Proof Using mainly identities (12), (13) and (14) we obtain

$$
\begin{aligned}
(1+ & +(1+x)(1+y)) z \\
\approx & (1+(1+x)(1+y) z) z \approx(1+(1+x)(1+y z) z) z \\
\approx & (1+(1+x)(1+y z)) z \\
\approx & 1+(1+(1+(1+x)(1+y z)) z) \\
\approx & 1+(1+(1+(1+x)(1+y z)) z)(1+(1 \\
& +(1+x)(1+y z) y z) \\
\approx & 1+(1+(1+(1+x)(1+y z)) z)(1+(1 \\
& +(1+x)(1+y z))(1+(1+y z))) \\
\approx & 1+(1+(1+(1+x)(1+y z)) z)(1+(1+(1+y z))) \\
\approx & 1+(1+(1+(1+x)(1+y z)) z)(1+y z) \\
\approx & 1+(1+(1+(1+x)(1+y z)) z(1+y z))(1+y z) \\
\approx & 1+(1+(1+(1+x)) z(1+y z))(1+y z) \\
\approx & 1+(1+x z(1+y z))(1+y z) \\
\approx & 1+(1+x z)(1+y z) .
\end{aligned}
$$


With the previous results in hand, we obtain now a simple proof of Theorem 5 .

Proof of Theorem 5 Let $\mathbf{R}=(R,+, \cdot, 0,1)$ be a uniquely representable generalized Boolean quasiring satisfying identity (12) and $\mathbf{L}=\left(R, \vee, \wedge,{ }^{\prime}, 0,1\right)$ denote the corresponding bounded lattice with an antitone involution. According to Lemma 7, $\mathbf{L}$ satisfies the identity $x \wedge x^{\prime} \approx 0$ and, according to Lemma $8, \mathbf{L}$ is distributive. Hence $\mathbf{L}$ is a Boolean algebra and therefore $\mathbf{R}$ a Boolean ring with unit. The rest of the proof is clear.

Acknowledgements Open access funding provided by TU Wien. This study was funded by the Austrian Science Fund (FWF), project I 1923N25, the Czech Science Foundation (GAČR), project 15-34697L, as well as by ÖAD, project CZ 04/2017.

\section{Compliance with ethical standards}

Conflict of interest The authors declare that they have no conflict of interest.

Ethical approval This article does not contain any studies with human participants or animals.

Open Access This article is distributed under the terms of the Creative Commons Attribution 4.0 International License (http://creativecomm ons.org/licenses/by/4.0/), which permits unrestricted use, distribution, and reproduction in any medium, provided you give appropriate credit to the original author(s) and the source, provide a link to the Creative Commons license, and indicate if changes were made.

\section{References}

Chajda I (1996) Pseudosemirings induced by ortholattices. Czechoslov Math J 46:405-411

Chajda I, Eigenthaler G (1998) A note on orthopseudorings and Boolean quasirings. Österr Akad Wiss Math Natur Kl Sitzungsber II 207:83-94

Chajda I, Länger H (2007) A common generalization of ortholattices and Boolean quasirings. Demonstr Math 40:769-774

Chajda I, Länger H, Mączyński M (2004) Ring-like structures corresponding to generalized orthomodular lattices. Math Slovaca $54: 143-150$

Dorninger D (1998) Sublogics of ring-like quantum logics. Tatra Mt Math Publ 15:75-83

Dorninger D, Länger H, Mączyński M (1997a) The logic induced by a system of homomorphisms and its various algebraic characterizations. Demonstr Math 30:215-232

Dorninger D, Länger H, Mączyński M (1997b) On ring-like structures occurring in axiomatic quantum mechanics. Österr Akad Wiss Math Natur Kl Sitzungsber II 206:279-289

Dorninger D, Länger H, Mączyński M (1999) On ring-like structures induced by Mackey's probability function. Rep Math Phys 43:499_ 515

Dorninger D, Länger H, Mączyński M (2000) Lattice properties of ringlike quantum logics. Int J Theor Phys 39:1015-1026

Dorninger D, Länger H, Mạczyński M (2001a) Concepts of measures on ring-like quantum logics. Rep Math Phys 47:167-176

Dorninger D, Länger H, Mączyński M (2001b) Ring-like structures with unique symmetric difference related to quantum logic. Discuss Math Gen Algebra Appl 21:239-253

Grätzer G (2011) Lattice theory: foundation. Birkhäuser, Basel

Länger H (1998) Generalizations of the correspondence between Boolean algebras and Boolean rings to orthomodular lattices. Tatra Mt Math Publ 15:97-105

Länger H, Mączyński M (2005) A Mackey-like approach to ring-like structures used in quantum logics. Rep Math Phys 56:413-419

Mackey GW (2004) Mathematical foundations of quantum mechanics. Dover Publications Inc, Mineola. ISBN 0-486-43517-2 